\title{
ADSORPTION IN MICROPOROUS MATERIALS: ANALYTICAL EQUATIONS FOR TYPE I ISOTHERMS AT HIGH PRESSURE
}

\author{
A. L. MYERS \\ Department of Chemical and Biomolecular Engineering \\ University of Pennsylvania, Philadelphia PA 19104 USA \\ E-mail:amyers@seas.upenn.edu
}

\begin{abstract}
Existing analytical equations for Type I isotherms such as the Langmuir equation and its modifications fail to describe experimental adsorption isotherms at high pressure. All of these equations predict that the amount adsorbed increases monotonically with increasing pressure. Experimental adsorption isotherms attain a maximum value in the amount adsorbed and then fall to zero. At the critical temperature of the gas, the maximum in the isotherm occurs at a pressure of about 10 bars and the zero occurs at a much higher pressure of several hundred bars. For subcritical gases, the maximum occurs at lower pressure and for supercritical gases the maximum occurs at higher pressure. The observed behavior of high-pressure adsorption isotherms can be predicted from the low-pressure (sub-atmospheric) portion by separating the Type I behavior expected for absolute adsorption from the experimental behavior observed for excess adsorption.
\end{abstract}

\section{Introduction}

Tabulated data for experimental adsorption isotherms are fitted with analytical equations for the calculation of thermodynamic properties by integration or differentiation. These thermodynamic properties expressed as a function of temperature, pressure, and composition are input to process simulators of adsorption columns. In addition, analytical equations for isotherms are useful for interpolation and cautious extrapolation. Obviously, it is desirable that the isotherm equations agree with experiment within the estimated experimental error. The same points apply to theoretical isotherms obtained by molecular simulation, with the requirement that the analytical equations should fit the isotherms within the estimated statistical error of the molecular simulation.

Type I isotherms [3] are characterized by an asymptotic approach to a saturation capacity with increasing pressure. This class of isotherms is most commonly observed for gases or vapors (water is an exception) adsorbed in zeolites or activated carbon. A typical set of Type I adsorption isotherms is shown in Fig. 1. Several questions may be asked about sets of isotherms like these. How is the saturation capacity measured? Is the saturation capacity a constant or does it decrease with temperature as suggested by Fig. 1?

Variation of the pressure $P$ with respect to temperature $T$ at constant loading $n$ is given by the equation [1]:

$$
\left[\frac{\partial \ln P}{\partial(1 / T)}\right]_{n}=-\frac{\bar{h}}{R}
$$

where $\bar{h}$ is the differential enthalpy of desorption (isosteric heat). This derivative of the adsorption isotherms in Fig. 1 along a horizontal line becomes ambiguous at high loading $(n)$. The differential enthalpy for this system measured by a calorimeter is plotted on Fig. 2. 
For type II isotherms with multilayer adsorption, the differential enthalpy for the second and higher layers approaches the enthalpy of condensation of the liquid. Does the differential enthalpy for type I isotherms approach some limit at saturation?

The intention of this paper is to seek answers to these questions.

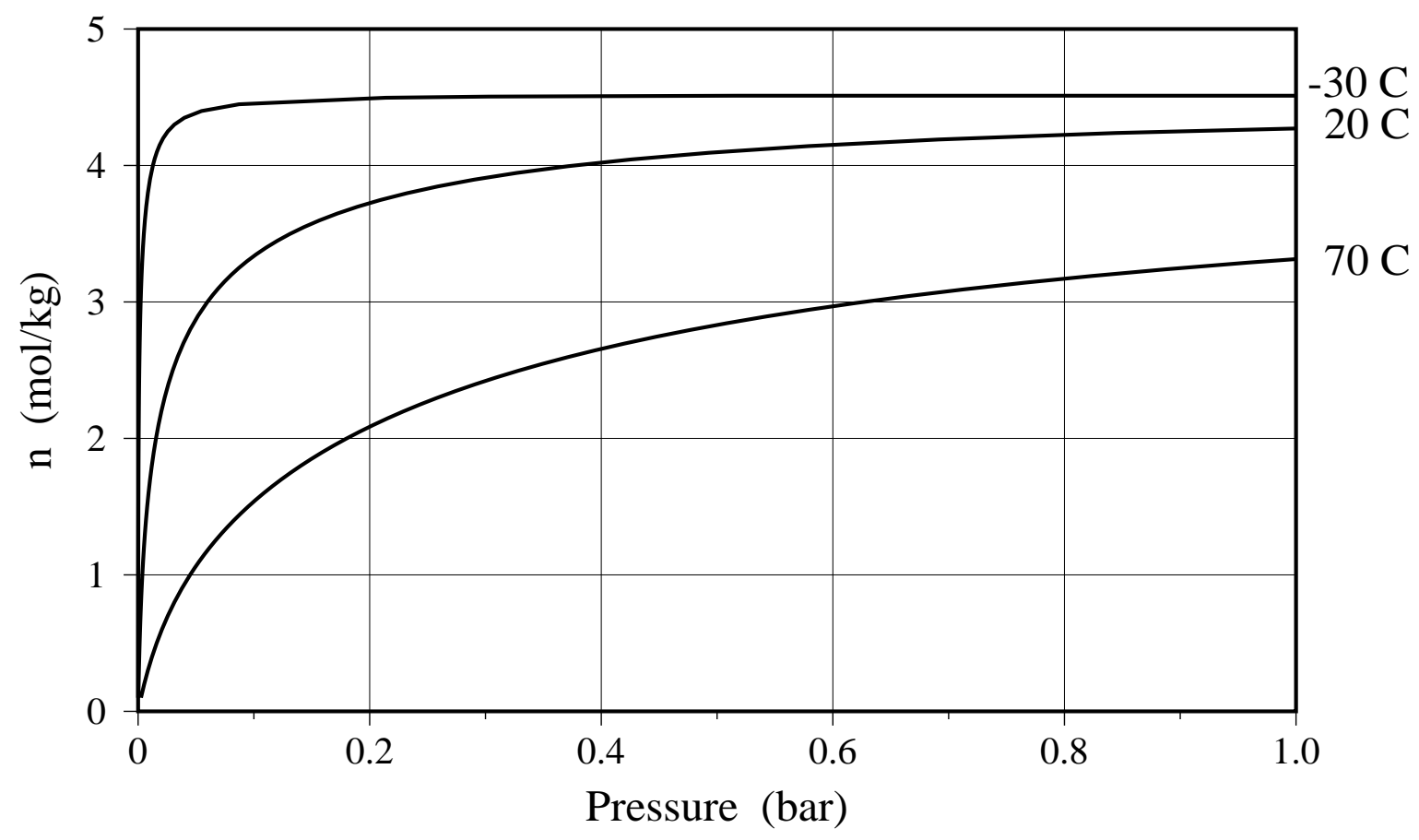

Fig. 1. Adsorption isotherms of $\mathrm{C}_{2} \mathrm{H}_{4}$ on $\mathrm{NaX}$ zeolite. The $20^{\circ} \mathrm{C}$ isotherm was measured experimentally [4]. The other two isotherms were calculated from Eq. (1) using the differential enthalpies in Fig. 2.

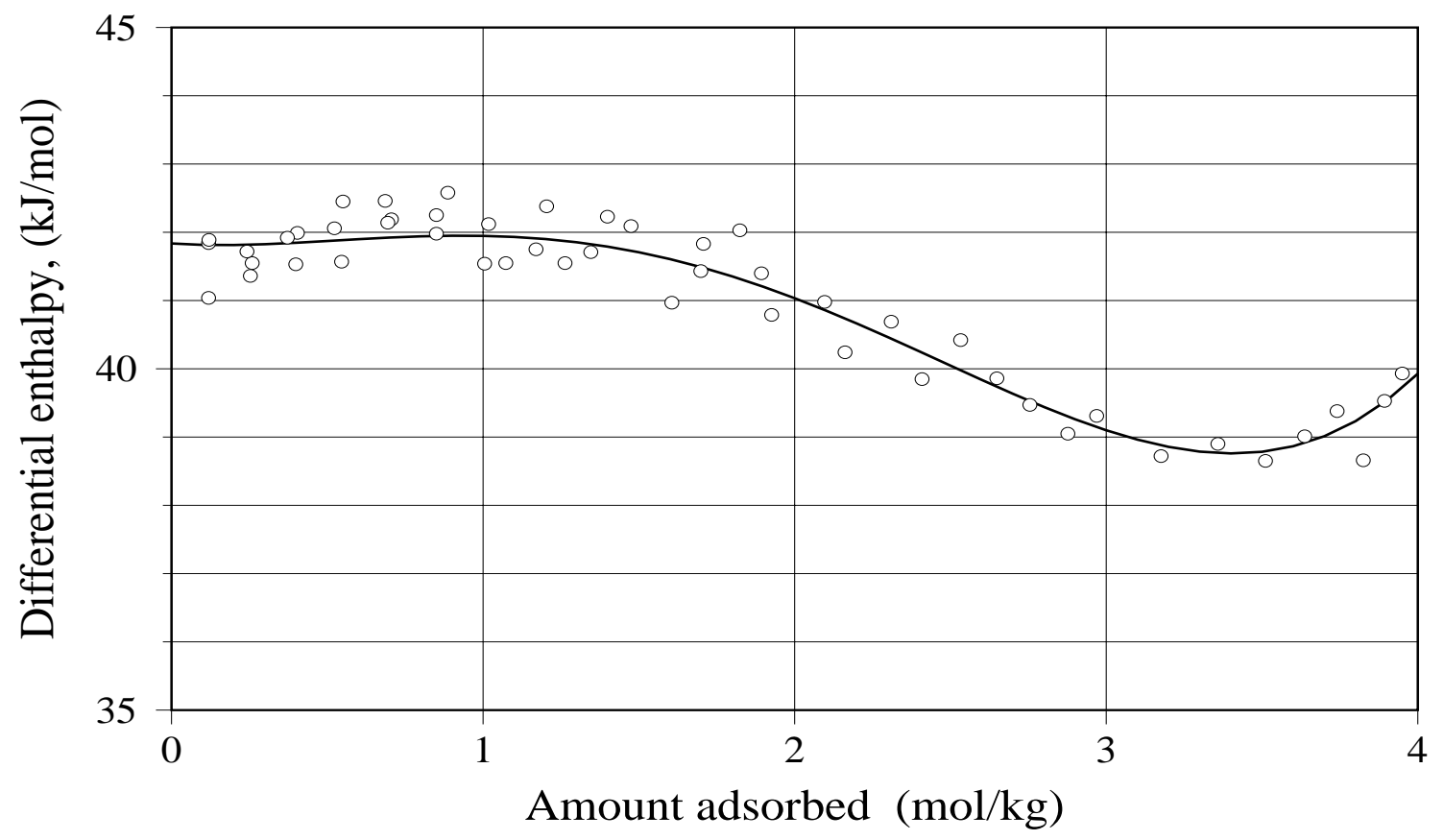

Fig. 2. Differential enthalpy of $\mathrm{C}_{2} \mathrm{H}_{4}$ adsorbed on $\mathrm{NaX}$ at $20^{\circ} \mathrm{C}$. Points are experimental calorimetric data [4]. Solid line is polynomial fit of data. 


\section{Adsorption isotherms}

A very useful equation for type I adsorption isotherms is [4]:

$$
P=\frac{n}{H}\left[\frac{m}{m-n}\right] \exp \left(C_{1} n+C_{2} n^{2}+C_{3} n^{3}+\cdots\right)
$$

where $H$ is the Henry constant, $m$ is the saturation capacity, and the $C_{i}$ are virial coefficients terminated after three or four terms, which are usually sufficient to fit adsorption isotherms over several decades of pressure. Unlike most adsorption equations, this equation is implicit in the pressure. This disadvantage is more than offset by the direct connection between the differential enthalpy and the temperature dependence of the virial coefficients. The main advantage of this equation is that it can be integrated analytically for the grand potential (spreading pressure), which is needed for mixture calculations as shown in this mixture section of this paper.

Experimental measurements yield excess adsorption; molecular simulations calculate absolute adsorption. The relationship between the two variables is given by:

$$
n^{e}=n-V_{p} \rho
$$

$V_{p}$ is the specific pore volume of the material; typical values are $200-400 \mathrm{~cm}^{3} / \mathrm{kg}$ for zeolites and up to $1000 \mathrm{~cm}^{3} / \mathrm{kg}$ for activated carbon. $n$ is the actual number of molecules contained in the micropores; the excess adsorption $n^{e}$ subtracts from $n$ the number of molecules which would have been present in the micropores at the bulk density in the absence of adsorption. The (oversimplified) case when absolute adsorption is described by the Langmuir equation and the gas obeys the perfect gas law $(\rho=P / R T)$ has been worked out in detail for the isotherms and thermodynamic functions (enthalpy, entropy, etc.) [2].

The key step in this development is the recognition that a Type I adsorption equation like Eq. (1) applies to absolute adsorption $n$. Absolute adsorption refers to the actual number of molecules present in the micropores and increases monotonically with pressure to an asymptote called the saturation capacity $m$. Experimental excess adsorption isotherms pass through a maximum and then decrease with pressure.

The adsorption isotherms in Fig. 1 may be considered absolute adsorption as a function of gas-phase fugacity $f$ of ethylene. For $P<1$ bar, the term $V_{p} \rho$ in Eq. (3) is negligible compared to $n$. Therefore a fit of these isotherms with Eq. (1) using constants reported previously [4] provides the absolute amount adsorbed $n(f)$. Given an equation for the absolute isotherm and the pore volume of faujasite $\left(340 \mathrm{~cm}^{3} / \mathrm{kg}\right)$, one can calculate the excess isotherms at high pressure. Taking the fugacity $f$ as an independent variable, the bulk properties of gaseous ethylene derived from the SRK equation [5] were used to determine the pressure $P$ and density $\rho=P /(z R T)$, where $z$ is the bulk compressibility factor of gaseous ethylene. The excess functions for three temperatures are plotted on Fig. 3. For sub-atmospheric pressure, the values of absolute and excess adsorption coincide. At higher pressure, the absolute adsorption approaches a saturation value $(4.534 \mathrm{~mol} / \mathrm{kg})$ while the excess adsorption passes through a maximum and eventually decreases to negative values at pressures above 100 bar. The isotherm for $-30 \mathrm{C}$ terminates at the dew point of ethylene vapor, 19.4 bar. The maximum excess adsorption decreases with temperature. At the pressure where the isotherms intersect, the temperature coefficient of adsorption is zero. Above this pressure, excess adsorption increases with pressure.

The maximum in the excess adsorption isotherm occurs at the point where the densities in the micropore and the bulk gas are increasing at the same rate with respect to pressure, so 
that an increase in pressure has no effect upon the amount adsorbed. Zero excess adsorption occurs at the pressure where the bulk and micropores densities are equal. Since the pore density at saturation is $\rho=m / V_{p}=(4.534 / 340)=0.0133 \mathrm{~mol} / \mathrm{cm}^{3}$, zero excess adsorption corresponds to the same value for the bulk density of ethylene. The pressures corresponding to this bulk density are 175 and 345 bar at $20^{\circ} \mathrm{C}$ and $70^{\circ} \mathrm{C}$, respectively. The density of $0.0133 \mathrm{~mol} / \mathrm{cm}^{3}$ inside the micropores is 1.7 times the critical density or 0.7 of the liquid density of ethylene at its normal boiling point $\left(-110^{\circ} \mathrm{C}\right)$. It is interesting that the micropore volume may be calculated from the bulk density at which excess adsorption is zero using $V_{p}=m / \rho$.

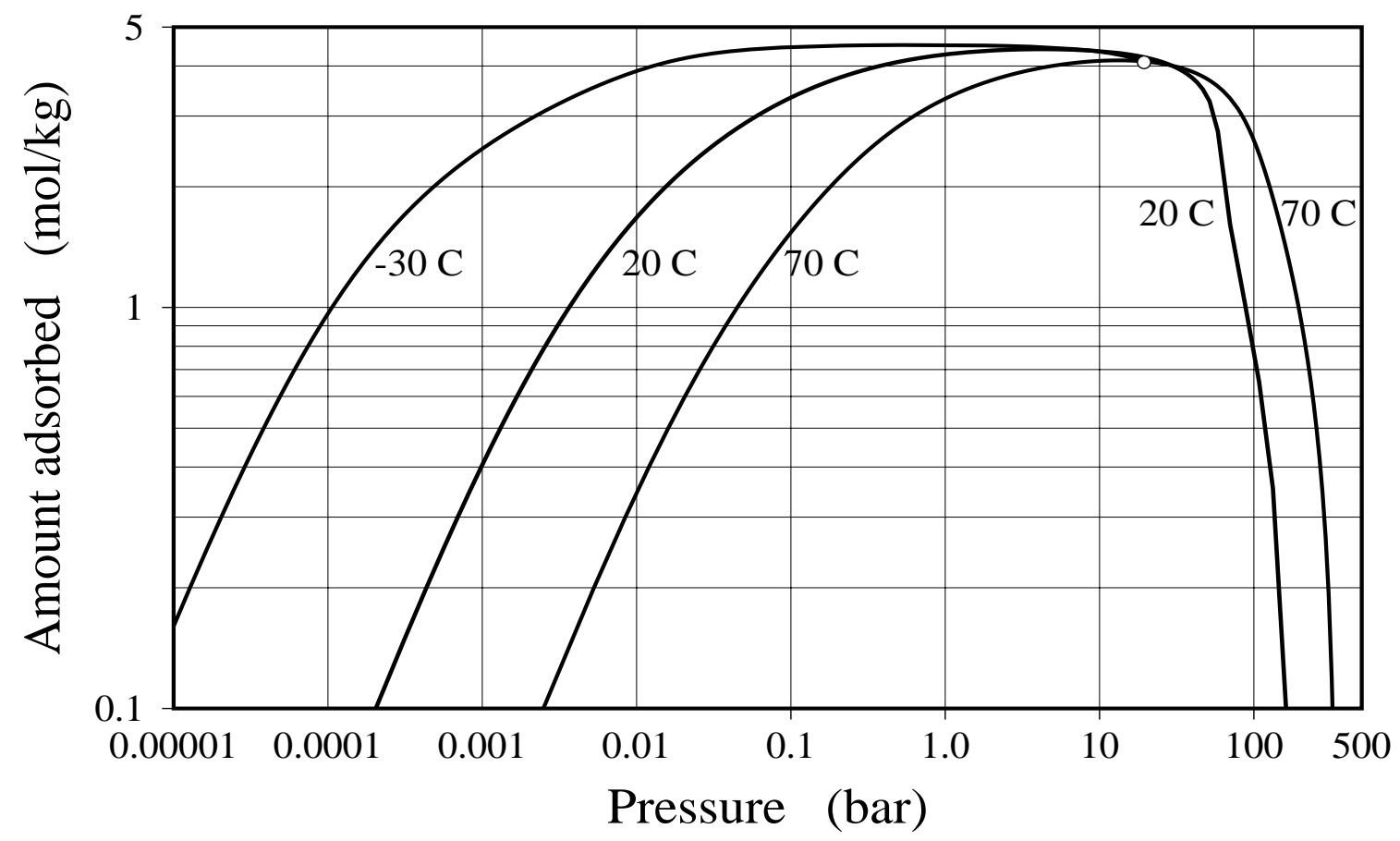

Fig. 3. Adsorption isotherms of $\mathrm{C}_{2} \mathrm{H}_{4}$ on $\mathrm{NaX}$. The $-30^{\circ} \mathrm{C}$ isotherm terminates at the dewpoint of ethylene vapor (19.4 bar)

The absolute adsorption isotherms for $-30^{\circ} \mathrm{C}$ and $70^{\circ} \mathrm{C}$ were calculated from the $20^{\circ} \mathrm{C}$ isotherm using the integrated form of Eq. (1) and the differential enthalpy plotted on Fig. 2. The reasonable approximation was made that the differential enthalpy is independent of temperature. No other assumptions were needed to calculate the excess adsorption isotherms on Fig. 3.

Examination of Fig. 1 answers the questions raised in the introduction about the determination of the saturation capacity. The saturation capacity for absolute adsorption cannot be extracted from supercritical isotherms measured at sub-atmospheric pressure such as the $70^{\circ} \mathrm{C}$ isotherm. Raising the pressure would bring the isotherm into the region where the difference between absolute and excess adsorption is no longer negligible. The Henry constant (the slope of the adsorption isotherm at the limit of zero pressure) is difficult to extract from sub-critical isotherms such as the $-30^{\circ} \mathrm{C}$ isotherm because the linear region occurs at very low pressure. The optimum temperature for the determination of both the Henry constant and the saturation capacity is near the critical temperature of the gas $\left(9^{\circ} \mathrm{C}\right.$ for ethylene), which is the $20^{\circ} \mathrm{C}$ isotherm on Fig. 1 . 


\section{Gas Mixtures}

The principles of phase equilibrium do not apply to excess adsorption variables at high pressure where the excess adsorption passes through a maximum. Under these conditions, the pressure is no longer a single-valued function of excess adsorption so that $n^{e}$ cannot serve as an independent variable for the determination of partial molar quantities such as activity coefficients. Additional complications which arise at high pressure are: (1) the selectivity for excess adsorption $\left(S_{12}=\left(n_{1}^{e} / y_{1}\right) /\left(n_{2}^{e} / y_{2}\right)\right)$ approaches infinity as $n_{2}^{e} \rightarrow 0$; and (2) the differential enthalpy of the $i$ th component has a singularity at the pressure corresponding to maximum $n_{i}^{e}$. For excess variables, the differential functions are undefined but the integral functions for enthalpy and entropy are smooth and well-behaved [1].

The principles of solution thermodynamics can be applied to absolute adsorption variables without any of these complications. For absolute variables, which arise naturally in molecular simulation, the pressure is a single-valued function of $n$, the differential functions exhibit no singularities, and the selectivity approaches a finite value as $P \rightarrow \infty$. Absolute adsorption may be determined experimentally by measuring excess adsorption in the usual way (volumetric or gravimetric method) at sub-atmospheric pressure where the difference between absolute and excess adsorption is negligible.

The fugacity equations are solved using absolute variables. As discussed previously, the single-gas isotherms at sub-atmospheric pressure provide the absolute isotherm in the form $f_{i}^{\circ}\left(n_{i}^{\circ}\right)$. Given the temperature of the isotherms, the independent variables are $P$ and $y_{1}$ in the bulk gas. For a binary mixture the fugacity equations are written:

$$
\begin{aligned}
& f_{1}\left(P, y_{1}\right)=f_{1}^{\circ}\left(n_{1}^{\circ}\right) \gamma_{1}\left(\psi_{1}\left(n_{1}^{\circ}\right), x_{1}\right) x_{1} \\
& f_{2}\left(P, y_{2}\right)=f_{2}^{\circ}\left(n_{2}^{\circ}\right) \gamma_{2}\left(\psi_{2}\left(n_{2}^{\circ}\right), x_{2}\right) x_{2}
\end{aligned}
$$

The fugacity $f_{i}=P y_{i} \phi_{i}\left(P, y_{i}\right)$ is determined from an equation of state (EOS) for the pure bulk gas. The adsorbed-phase activity coefficients are functions of the grand potential $(\Omega)$ and composition, where $\psi \equiv-\Omega / R T$. The form of Eq. (2) allows the grand potential to be calculated analytically [4]:

$$
\psi(n)=-m \ln \left(1-\frac{n}{m}\right)+\frac{1}{2} C_{1} n^{2}+\frac{2}{3} C_{2} n^{3}+\frac{3}{4} C_{3} n^{4}+\cdots
$$

The standard-state properties are measured at equal values of the grand potential:

$$
\psi_{1}\left(n_{1}^{\circ}\right)=\psi_{2}\left(n_{2}^{\circ}\right)
$$

Given a bulk EOS to determine the fugacity functions and noting that $\left(x_{1}+x_{2}\right)=\left(y_{1}+y_{2}\right)=$ 1, the three Eqs. (4), (5), and (7) can be solved for the three unknowns: $n_{1}^{\circ}, n_{2}^{\circ}$, and $x_{1}$. Following methods described previously [4], the absolute total $(n)$ and individual amounts adsorbed $\left(n_{1}, n_{2}\right)$ are calculated from the standard-state values $n_{1}^{\circ}$ and $n_{2}^{\circ}$. Finally, using an EOS to determine the bulk density $\rho\left(P, y_{1}\right)$, the absolute variables are converted to experimental excess variables by [1]:

$$
n_{i}^{e}=n_{i}-V_{p} \rho y_{i}
$$

Excess isotherms are shown on Figure 4 for binary mixtures of ethylene and ethane adsorbed on $\mathrm{NaX}$. Both curves are for $20^{\circ} \mathrm{C}$ and a vapor composition of 10 mole percent ethylene. The individual excess isotherm for ethylene is the difference between two isotherms. 
Ethylene is preferentially adsorbed and the individual isotherm for ethane reaches a maximum at a pressure of 3 bar. The individual isotherms show the same behavior as the single-gas isotherms shown on Figure 3: a maximum value in the amount adsorbed followed by a steep decline to zero.

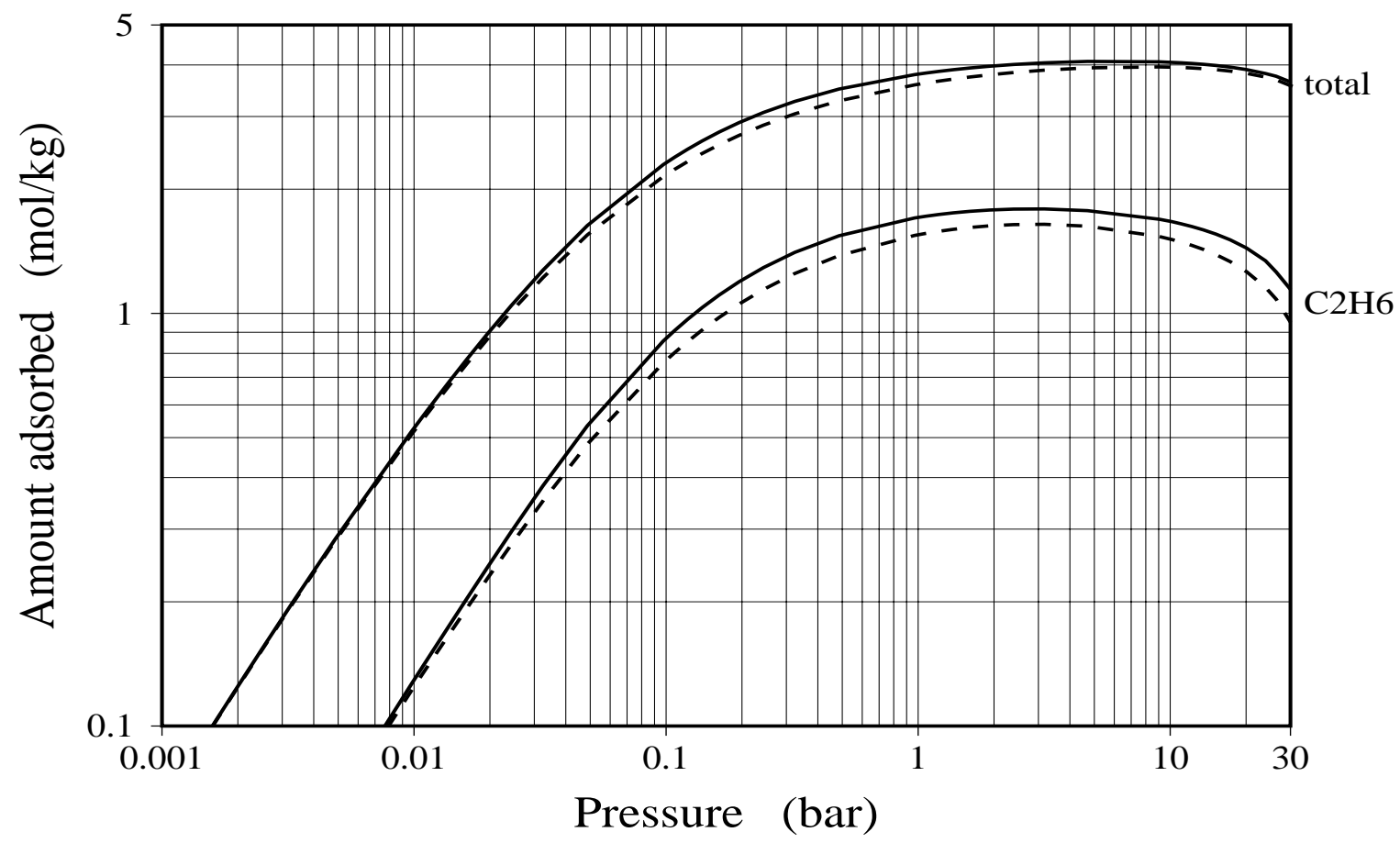

Fig. 4. Adsorption of mixtures of $\mathrm{C}_{2} \mathrm{H}_{4}$ and $\mathrm{C}_{2} \mathrm{H}_{6}$ on $\mathrm{NaX}$ at $20 \mathrm{C}$ for 10 mole \% ethylene in gas phase. The individual isotherm for $\mathrm{C}_{2} \mathrm{H}_{4}$ is the difference between the total and the individual isotherm plotted for $\mathrm{C}_{2} \mathrm{H}_{6}$.

The solid lines on Figure 4 take into account the nonideal behavior of adsorbed mixtures of ethylene and ethane in $\mathrm{NaX}$. This system is highly nonideal because of the interaction of the quadrupole moment of ethylene with the sodium cations of $\mathrm{NaX}$. Activity coefficients at infinite dilution are unity at the limit of zero pressure and 0.27 at high pressure. The dashed lines on Figure 4 were calculated for an ideal adsorbed solution (IAS) and the resulting error in the individual isotherm for ethane at 30 bar is $20 \%$.

\section{Discussion}

The high pressure adsorption of single gases and mixtures can be predicted from the low pressure (sub-atmospheric) data for the same systems. The optimum temperature for measuring the adsorption of single gases is near their critical temperature where both the Henry's constant and the absolute saturation capacity can be determined accurately.

The absolute adsorption isotherm as a function of gas-phase fugacity is obtained directly from molecular simulations based on the grand canonical Monte Carlo (GCMC) method. Since the difference between absolute and excess adsorption is negligible at sub-atmospheric pressure, the low-pressure portion of the absolute isotherm can also be determined from experiment. Eq. (2) is suitable for extrapolating the absolute isotherm from low to high pressure and Eq. (3) provides the conversion to excess adsorption. Experiments are needed to test these predictions of adsorption at high pressure. 


\section{References}

[1] Myers, A. L., A.I.Ch.E. J., 2002, 48, 145.

[2] Myers, A. L., and Monson, P. A., Adsorption in porous materials at high pressure: Theory and experiment, Langmuir, 2002, in press.

[3] Ruthven, D.M., Principles of Adsorption and Adsorption Processes, Wiley Interscience, New York, 1982.

[4] Siperstein, F.R. and A.L. Myers, A.I.Ch.E. J., 2001, 47, 1141.

[5] Smith, J. M., Van Ness, H.C., and Abbott, M.M., Introduction to Chemical Engineering Thermodynamics, 6th Edition, McGraw-Hill, New York, 2001. 